\title{
Infrared Thermography and NDT: 2050 Horizon
}

\author{
by F. Khodayar*, S. Sojasi* and X. Maldague*
}

* Computer Vision and Systems Laboratory, Electrical and Computer Engineering Dep., Université Laval, Quebec City (Quebec), G1K 7P4, Canada, fariba.khodayar.1@ulaval.ca,saeed.sojasi.1@ulaval.ca,Xavier.Maldague@gel.ulaval.ca

\begin{abstract}
Society is changing fast, new technologies and materials have been developed in which require new inspection approaches. Infrared Thermography (IRT) has emerged in the recent years as an attractive and reliable technique to address complex Non-destructive (NDT) problems. Companies are now providing turn-key IRT-NDT systems, but the question we ask now is "What is next?". Even though the future is elusive, we can consider the possible future developments in IR NDT.

Our analysis shows that new developments will take place in various areas such as: acquisition, stimulation, processing and obviously an always enlarging range of applications with new materials which will have particular inspection requirements.

This paper presents the various developments in the field of IRT which have evolved to lead to the current situation, and then examines the potential future trend in IRT-NDT.
\end{abstract}

\section{Introduction}

Infrared thermography (IRT) is an attractive method to analyze the thermal information obtained from a specimen. This technique detects energy emitted from the specimen under investigation and converts it to temperature variation. The output is an image of temperature variation from the specimen. Infrared refers to the radiations located between visible and microwave in electromagnetic bands [1-3]. Generally, infrared bands are divided into three parts, mid-wave-infrared (MWIR), long-wave-infrared (LWIR), and far-infrared (FIR), which are respectively from 0.75 to $3 \mu \mathrm{m}, 3$ to $5 \mu \mathrm{m}, 8-12 \mu \mathrm{m}$, and 50 to $1000 \mu \mathrm{m}$ [1]. Infrared radiation was unknown until more than 200 years ago, when Herschel conducted the first experiment with a thermometer. He built a crude monochromator in which a thermometer was used to detect radiative energy under sunlight [4].

Non-destructive testing (NDT) is a method for testing materials without destroying them. More specifically, these methods are used to evaluate and inspect processes and materials so as to identify the differences in characteristics, and components for discontinuities. The NDT methods are attractive and useful to determine ductility and impact resilience, fatigue strength, fracture, flaws, abnormal operations, etc. Non-destructive testing methods keep the function of mechanical components safe, reliable, and cost-effective. This technique is widely used in science and industry, especially since it has no damaging effect on materials [5, 6].

Infrared thermography is one of the useful techniques that has been used in Non-destructive testing. Using infrared technology has many advantages such as the fact that it is a non-contact method, non-destructive and fast technique, which does not emit any harmful radiations, etc. [7]. The current paper will offer insight into the future developments of IR thermography based on an overview of literature from the past to the present, including IR applications, progress and processing, and IR markets. The vision of IRT and future trends in IRT will be examined based on an overview of the developments which have led to the current situation in the field. Infrared thermography strives towards using equipment offering high performance, high accuracy and low cost. Infrared thermography may soon involve the use of robots in dangerous environments, which will be discussed as well. The new technologies and advances in IR thermography have created new markets such as: intelligent building, automotive and environment control [8].

\section{Short history of infrared technology}

After the discovery of infrared light in 1800, and the development of important laws such as the Stefan-Boltzmann's Law and Planck's law [9-11], the "revolution" in thermal imaging has really been the emergence of uncooled IR arrays based on thermal detectors with the ability to work at room temperature. Thermal detectors had been used before in scanning imagers, but they had a slow response. With a low bandwidth, these thermal detectors were useful for example for scanning 2D electronic arrays [4]. Nowadays arrays of thermal detectors achieve interesting capabilities hence opening new perspectives of use.

The other main technology is infrared photodetector technology which underwent rapid development in the last decades of the $20^{\text {st }}$ century. Two IR systems were especially well developed: indium antimonide (InSb)- and mercury cadmium telluride (HgCdTe-based) detectors. They are now commercially available and their developments have continued to progress over the last few years [12]. Molecular beam epitaxy (MBE) was used in the growth of Mercury cadmium 
telluride (MCT) detectors in 2000. At that time, most of the published research covered two main subjects: MCT on silicon substrates and Long-wavelength infrared (LWIR) detectors. LWIR detection was an extension of Short-wavelength infrared (SWIR) and Mid-wavelength infrared (MWIR) [12 13]. Towards 2010, infrared community users have devoted more attention to portable equipment in the field of active thermography [13, 14]. Ongoing research has improved the performance of room-temperature devices in terms of improved detectivity [2, 9].

\section{Infrared detectors}

An infrared detector is sensitive to infrared radiation energy and reacts to this energy and converts it into a quantifiable form. A review of IR detectors indicates that there has been three significant developments in IR detectors: the first one dealt with scanning systems, the second one was staring array systems and the third involved multicolor detectors [15]. Some consequential parameters which affect the performance of infrared detectors are signal to noise ratio, specific detectivity $D^{*}$, noise equivalent temperature difference (NETD), spectral response and acquisition time [16, 17]. There are different types of infrared detectors, which can be classified according to different criteria such as wavelength, sensitivity, power dissipation, and bandwidth [18]. A certain type of classification, divides infrared detectors into two general types: the first type is thermal or bolometer detectors, which are low-cost and do not depend on wavelength, but they have inferior performance with high production volume. Their cost is dependent on the cooling system to a significant extent (they are often temperature stabilized using Peltier elements). The second type is the quantum type or the photon detector that is dependent on wavelength, requires expensive cryogenic cooling mechanisms and has a lower production volume with high cost. Their sensitivity and response time are typically higher than bolometer detectors [2, 16, 17, 19, 21].

\subsection{Current infrared detectors and future developments}

Nowadays, IR detectors are based on three important technologies; one of them is Mercury-cadmium-telluride (MCT), an expensive technology due to the use of CdZnTe. The Indium antimonide (InSb) detector is another type of detector, which is photovoltaic, and where the indium antimonide requires periodic recalibration. InSb is a function of thickness near the band edge photons and it has 100\% internal quantum efficiency [2, 20, 21]. Quantum-well infrared photodetectors (QWIPs) represent another existing IR technology which is dependent on the wavelength range, and can cover a wide range of wavelengths. Since this technology operates at low temperature and requires cryogenic systems, it is expensive: but in comparison with MCT, it has a lower cost [2, 20, 22].

Recent research on infrared thermal detector technologies has focussed on the following goals: lower cost, higher performance, larger detectivities, portable devices, the use of focal plan array to obtain the highest number of pixels, the ability to work at higher temperatures and elimination of cryogenic cooling [2, 11, 20, 21 23--25]. One of the new technologies is quantum dot based infrared detectors (QDIPS), which perform well in the MWIR as well as the LWIR range [26]. They are very similar to QWIPs but with some advantages such as: normal incident detection, low dark current, high absorption coefficient, ability to work at higher temperatures, multicolor detection, and lower cost [2, 20, 21, 27, 28].

Looking to the future, infrared thermal detector technologies will continue to aim for higher sensitivity, higher efficiency and better performance at a lower cost. These detectors will be further developed to increase their operating temperature, reduce power dissipation and eliminate cooling system and cryogenic components. Future work will be directed towards increasing pixel density, embedding detectors with smart algorithms, improving response time, increasing spatial resolution, reducing non-uniformity and pixel size, as well as enabling multispectral capabilities. One important issue is however the (huge) investment required by the industry. In that sense we might see a reduced pace to performances related to the necessity to first provide a return on investments with the current technology.

\section{Smart sensors}

Smart sensors are defined as sensing elements with embedded intelligence [29]. They can perform many functions such as: data conversion, logic function, decision making, bidirectional communication, signal detection, signal processing, and data interpretation [29, 30]. Applications of smart sensors are divided into the following groups: optical sensors, infrared detector arrays, accelerometers, multisensory integration, etc. [17, 30].

Infrared thermography involves very small signals, which are very sensitive to noise [17, 30]. Infrared temperature measurement could be combined with digital technology through smart infrared sensors [31]. Smart sensors have several advantages such as: high reliability, simplified design, high performance, minimum cost and small size. Smart Sensing has led to considerable developments in consolidated Solid State Very Large Integration Microcircuits VLSI including MEMs technology [32] and in the new emerging nanotechnology. Nanotechnology allows sensors to be smaller, smarter and less costly [32]. These new sensors are being developed with Carbon nanotubes, graphenes and nanoparticles [26]. Future trends in thermal detectors indicate an increasing usage of intelligent sensors. Smart sensors will be able to extract signals, conduct signal processing and select information. The Smart sensors of the future will provide higher accuracy, higher performance with increased speed at a lower cost [17, 33, 34]. 


\section{Multi-band detector}

A critical issue for capability development of active and passive remote sensing in the UV to far-infrared spectroscopy is the development of multi-band detectors [35]. Multi-band detectors are used in numerous applications such as medical imaging, military, remote sensing, etc. A multi-band detector is formed of a stacked arrangement of different materials in which the longer wavelength detector has been located below the shorter wavelength detector [35]. The shorter wavelength detector absorbs the shorter wavelength, and the longer wavelength detector absorbs only the longer wavelength [35]. In other words, the desired wavelength band is absorbed by a suitable detector and the rest of the spectrum, without much losses, is transmitted to and absorbed by other appropriate detectors. Inspecting a component at various wavelengths provides more information about it and its behavior under test conditions. In that sense, multi-band detectors enable a more complete inspection, especially when coupled with data fusion of the recorded signals.

\section{Terahertz technology}

Recent works in optical NDT technology have improved sensitivity, the accuracy of detection, signal multiplexing, in addition to finding solutions for eliminating electromagnetic interference. Among these advances, the term "terahertz" (THz) has been used to refer to a part of the electromagnetic spectrum which is located between infrared light and microwaves (frequency range: $300 \mathrm{GHz}$ to $3 \mathrm{THz}$ with corresponding wavelength range: $1 \mathrm{~mm}$ to $100 \mu \mathrm{m}$ ). There are various THz systems which can be divided into two principal kinds: continuous wave $(\mathrm{CW})$ and picosecond pulses. $\mathrm{CW}$ could be generated by two near infrared lasers of adjacent wavelengths which are spatially overlapped. This technique has some advantages such as: high resolution, spectral selectivity, and superior SNR values [36]. Femtosecond lasers generate Pulsed terahertz radiation. The ultrashort (ultrafast) laser pulses produce a fast current transient. This laser emits electromagnetic waves in the terahertz range [37]. In the last few years, terahertz detectors and sources have been developed considerably and used as one of the new non-destructive testing technologies.

By analyzing changes in the $\mathrm{THz}$ signal, the internal structure of the object can be determined and the defects can be identified [38-40]. In comparison to other NDT techniques, $\mathrm{THz}$ has the particular advantage of being able to detect internal defects in non-metallic materials. THz radiation penetrates clothing and many other opaque materials; it is also selectively absorbed by water and organic substances. These unique qualities make $\mathrm{THz}$ radiation interesting and informative. When a source with a fixed-frequency and a single detector is applied, the continuous wave (CW) terahertz does not have the capability of providing any depth, frequency-domain or time-domain information. $\mathrm{CW}$ imaging is less complex than a pulsed $\mathrm{THz}$ system, since the CW imaging does not require a pump probe system, and also it is a compact, simple system [40]. Terahertz is safe to use on living organisms (non ionizing radiation) and has a shorter wavelength and higher spatial resolution than microwave radiation. It is increasingly been used in a large range of fields such as spectroscopy, medicine, non-destructive testing, chemistry, agriculture, food industry, materials science, biology and pharmacy [40,-45].

This technique has been used through fiber stretchers with kilohertz acquisition rates [40], and also, with porous polymer fibers designed to guide terahertz radiation. One of the particularities of $\mathrm{THz}$ detection methods is using semiconductor quantum dot detectors to detect a single $\mathrm{THz}$ photon. Two detection technologies which use $\mathrm{THz}$ cameras have been improved in the recent years: 1) Plasma-wave detectors and arrays 2) Un-cooled bolometer detectors and arrays [46]. In the last few years, THz has been more and more applied in the field of NDT, for example in the aerospace industry for defect detection, and stress damage evaluation in airplanes and spacecrafts [38].

Some companies such as Terasense and NeTHIS are developing THz technology and reaching interesting performance, simply, and in a less expensive fashion. Terasense proposes plasmonic detectors in the terahertz range which have high sensitivity and low cost [47]. The detector in the array measures the dispersion of magnetoplasma and plasma excitations (resonance) in an electron system whose edges are formed by a voltage applied to a metallic gate [48]. For generation, they use an IMPATT (IMPact ionization Avalanche Transit-Time) diode which is a high-power diode used in microwave electronics and sub-THz devices (frequency range $3-400 \mathrm{GHz}$ ). Another example of innovation is the TeraCam camera which is produced by NeTHIS. This camera is a Multispectral camera (IR and TeraHertz). The TeraCam has been designed for laser beam metrology, 2D and 3D imaging, and Non Destructive Testing of insulating materials [49]. NeTHIS uses a transfer process in which THz radiation heats a surface imaged by the IR camera, the IR camera being fitted with a "converter" kind of adapter.

In the future, $\mathrm{THz}$ technologies will strive toward cost reduction, increased spatial resolution, with real-time capability imaging [39, 40, 45, 50]. The development of $\mathrm{THz}$ technology will continue as the technology evolves to reach a certain threshold, upon which the mature technology will most certainly attract considerable attention [51].

\section{Data processing algorithms for Thermal Infrared NDT}

One of the most important steps in IR imaging is data processing to extract the desired information. Data processing involves a series of advanced mathematical operations which are performed on the data to transform it into useful 
information. IR images are strongly influenced by vignetting, spectrometer drift, radial distortion, environment reflections, emissivity variation, non-uniform radiation, fixed pattern noise, etc. [52, 53]. The purpose of signal processing is to eliminate unwanted signals and noise [54]. There are many data processing algorithms and the selection of the method depends on the objectives of the research. Some of the most useful techniques are [54-67]: Thermal signal reconstruction (TSR), Differential absolute contrast (DAC), Pulsed phase thermography (PPT), Principal component thermography (PCT), Partial least square thermography (PLST) and Supervised principal component analysis (SPCA). Thermal infrared NDT data processing techniques have some advantages and limitations such as defect detection enhancement on one hand, but sometimes exhibit slow computing or require interactions with an operator to select algorithm parameters on the other hand (ex: selection of a non-defect area which could affect final results). The performance of these techniques can be evaluated quantitatively with methods such as: signal to noise ratio, Tanimoto criterion and Probability of detection(PoD). PoD is very important for industry since it is a quantitative accepted measurement of reliability. In the future, these techniques will likely see enhancements while others will be proposed (such as PLST which has been known in the field of economics for decades but was only proposed recently in IR-NDT).

Quantitative extraction of information is still on the agenda in IRT and has seen considerable efforts. It is expected that advances in that direction will be achieved through the coupling of IRT image processing as described above coupled with appropriate thermal models of the studied processes. An example was shown recently using a thermal quadrupole based model whose parameters were adjusted recursively by non-linear least square minimization enabling the computation of local thermal diffusivity [68].

\section{IRT applications: NDT and more}

In the few past years, infrared thermography has been considerably developed and has found applications in analysis, detection in various fields such as medicine, industry, etc. Some of the most important applications of infrared thermography are listed below [69] 70]:

$\checkmark$ Infrared thermography is widely used in NDT, both in industry and R\& D, to detect a wide variety of defects. NDT and Condition Monitoring (CM) are tools used to find defects in materials and machinery, to investigate and control materials and processes without causing any damage. The quality of engineering materials and the safety of engineering structures can be evaluated with NDT. A new trend is that NDT can also be used virtually in industry to ensure that final products do not have any defects due to possible difficulty of assembly on the production line. Virtual NDT inspection proceeds at the design stage of the component enabling the anticipation of how (well) a given part could be checked by NDT [71]. This is very important in airplanes and engines, nuclear plants, ships and satellites, etc. [70].

$\checkmark$ Infrared images have a large variety of applications such as: in medicine (to observe blood flow changes in the skin, in treatment of high blood pressure, arthritis, heart failure, chronic fatigue, stress, toxicity, etc.), in the military field (military surveillance, target tracking, night vision, etc.), in industry (analyzing the thermal efficiency of processes and machinery, inspection in industry, monitoring the environment, etc. ), in civil engineering (to detect defects, to determine moisture content, to conduct quality control of buildings, etc.), in remote sensing (to predict climate change, to locate materials, etc.), in chemical industries (to identify molecular structure and composition and their emission and absorption, etc.) [69, 70] among other applications. With the falling price of IR cameras and computers even more applications of IRT will emerge, in any field where a non-contact change of temperature witnesses a phenomenon of interest.

\section{Recent and new infrared technologies}

Future IR technologies strive to develop detectors which are portable and have a high sensitivity, lower cost, high resolution, etc. [26]. Some of the new systems and devices are described below.

The first is the Heat-Wave, which is a $3 D$ device combined with a thermal camera, color camera and a range sensor (RGB-D camera and single additional thermal camera). The fact that the Heat-Wave is a light-weight thermal infrared camera is a great advantage. All sensors in this device have close format and are mounted on an ergonomic handle for portable deployment [72]. The various viewpoints recorded improve the accuracy. The Heat-Wave can be used in different applications such as: energy efficiency monitoring, energy losses, mechanical and electrical assessment, construction monitoring, fire detection, first responders and non-invasive medical diagnosis, etc.[72]-74]. This system can also be used in NDT, since it can locate sources of energy losses in buildings and can identify faulty components with abnormal temperatures in industry [72-74]. In these applications the advantage is obviously the projection of thermal data on the 3D shape of the object, enabling a better understanding of the infrared signature. 


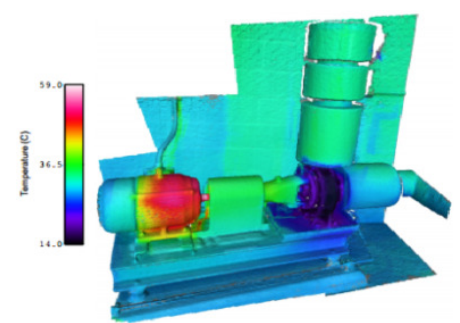

(a)

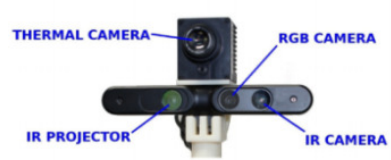

(b)

Fig. 1. (a) $3 D$ thermal map of a chiller water pump system using the rainbow color palette. (b) HeatWave $3 D$ thermography device [72-74]

Other interesting systems are small low cost IR detectors [25]. One of the new areas of interest in infrared technologies is the panoramic IR technology, which is designed to capture 360 degree images [25]. In the future, this technology could be used in low cost and low resolution systems in vehicles and boats to increase safety, especially at night or in conditions of bad visibility (smoke conditions, etc.). High-resolution systems which are more expensive would be suitable for use in ships and submarines. In a panoramic system, the frame rate is very important [25]. If the frame rate of the scanned system which rotates to provide a photo is too slow, the camera would not be able to recognize the target and enable the tracking [25]. In some instances, a "pyramidal-shaped" mirror can be used in front of the IR camera to acquire a $360^{\circ}$ image at once. Such images are however distorted and need correction before being displayed. This technology can be very useful in the field of non-destructive testing, in the field of medicine, because it can be applied to cylindrical objects and offers a global internal thermal view.

Robots have recently been combined with other infrared technologies, in a variety of applications (see figure 2). Robots can be used in automated NDT, in complex geometries, and in hazardous areas [75]. In the future, the robotic technology will produce humanoid robots, similar to the one shown in figure 2(b) which could hold an infrared camera in one hand and a heat source in the other, and inspect a variety of specimens. In the next 25 years, NDT robots will be equipped with a thermal camera, a color camera and a range sensor in their "eyes". The robot's vision system will detect the defects with the help of an analytical system using data processing algorithms, all embedded in the robot's "brain" [76]. This is in fact the new trend of the so-called Industry 4.0 and the Internet of things (IOT), seen by some as the next industrial revolution [77]. IOT is the network of physical objects or "things" including robots, IR cameras embedded with electronics, software, sensors and connectivity to enable it to achieve greater value and service by exchanging data with the manufacturer, operator and/or other connected devices [78]. IR NDT will exploit these novelties as well, as explained briefly below.

Today, microbolometers arrays are becoming more popular. Their launched price was over ten thousand dollars, but elementary complete systems can be purchased for several hundred dollars in 2015 [25. Such systems have been used in thermography, automotive applications, and other fields for a few years already, and low-cost arrays have increased the popularity of thermal imaging (example FLIR Lepton - see below - offered in kits on popular microcontroler platforms such as Raspberry Pi or Arduino). Another recent example is the FLIR ONE which is a thermal imager for smartphones that was unveiled in January 2014 (see figure 2 (d)). Its cost is $\$ 350$ and includes a visible camera and a microbolometer camera that is presented on the smartphone screen [25]. The small thermal camera in this case is a very low-cost microbolometer-based core (called Lepton). The Lepton is an 80-by-60-pixel array with 17- $\mu \mathrm{m}$ pitch detectors. Its sensitivity band is in the range of 8 to $12 \mu \mathrm{m}$ (LWIR) and the fitted lens has a 50-degree horizontal field of view [25]. FLIR ONE can be used to locate warm or cold air leaks in homes, heat losses through windows and insulation, identify moisture in buildings, detect overloaded electrical connections, locate pipes behind walls and under floors and control radiant floor heating and even more applications depending on the user's imagination [79]! 


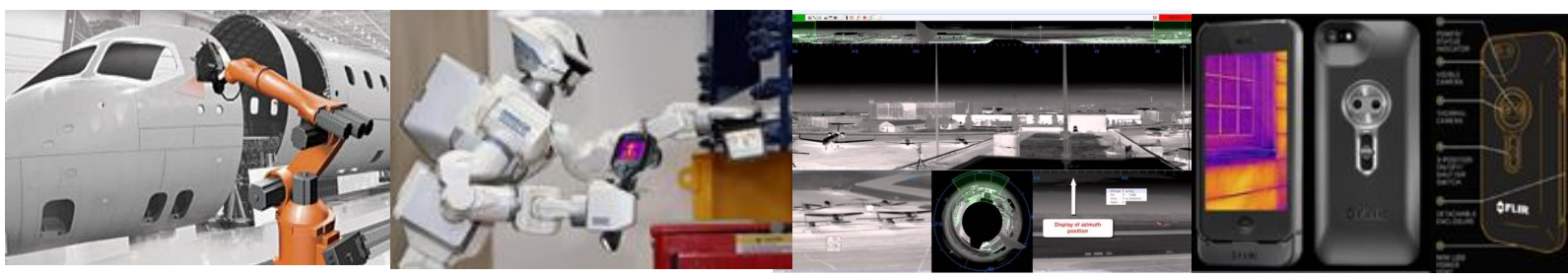

(a)

(b)

(c)

(d)

Fig. 2. ( $a$ and b) The robots in NDT, (c) Panoramic IR image, (d) FLIR One infrared camera (image courtesy of FLIR) [25, 79]

The Seek thermal camera is another thermal imaging camera which uses more than $32,000(206 \times 156)$ thermal pixels to create a IR image displayed on iOS and android devices (see figure 3). This type of camera is very small and has a plug adaptor for USB ports. This camera does not require batteries or special cables. The software is similar to that of Flir One. This camera has a spot meter function to display surface temperatures [80].

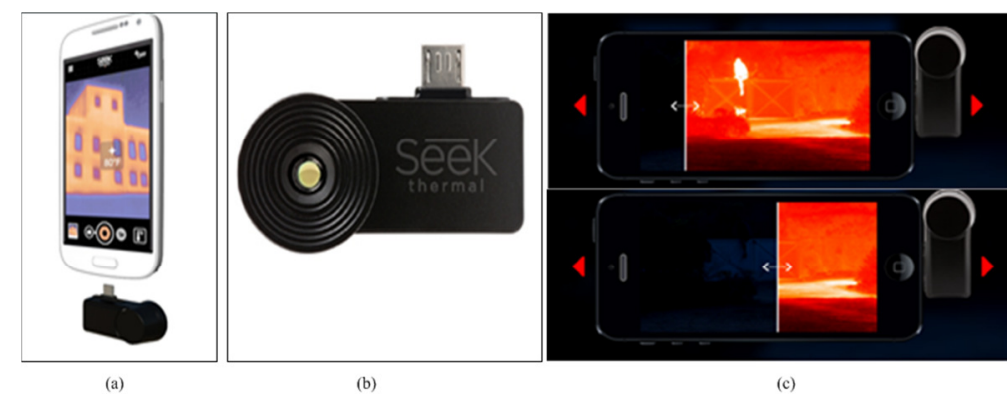

Fig. 3. Figure 3: Seek thermal camera (courtesy of Seek Thermal) [80]

The reduction in price of IR sensors (and in the price of more powerful pocket size computers) will enable the emergence of "stand-alone dedicated IR systems". For example, PC computers are now available on a USB key (ex: Intel's "Compute Stick". The same for IR sensors with an USB interface: a full system including Bluetooth, WI-FI is thus available for a minimum hardware cost of less than 350 \$ (PC: $149 \$+$ IR: 199 \$). The price will most likely continue to decrease in the years ahead. Such USB-IRT-PC could be loaded with IR image processing software for special applications such as in monitoring overheating in electric cabinets, detection of subsurface defects on production lines, etc. WIFI could be used to communicate automated diagnosis to a central unit and, in case of IR-NDT, activate stimulating sources (such as pulse heating). In the future, at even lower cost, size and weight, these systems could even be deployed permanently inside critical (electrical cabinets, etc.) components for continuous check-up following the previously discussed IOT trend.

All technologies related to infrared are developing very fast. Today with changes in detectors, camera sensors, and infrared systems, it is important to have high resolution, high performance, and low cost systems. These goals will become even more obvious in the future as illustrated in figure 4 which shows the expected reduction in infrared camera cost accompanied by an increase in pixel capability. 


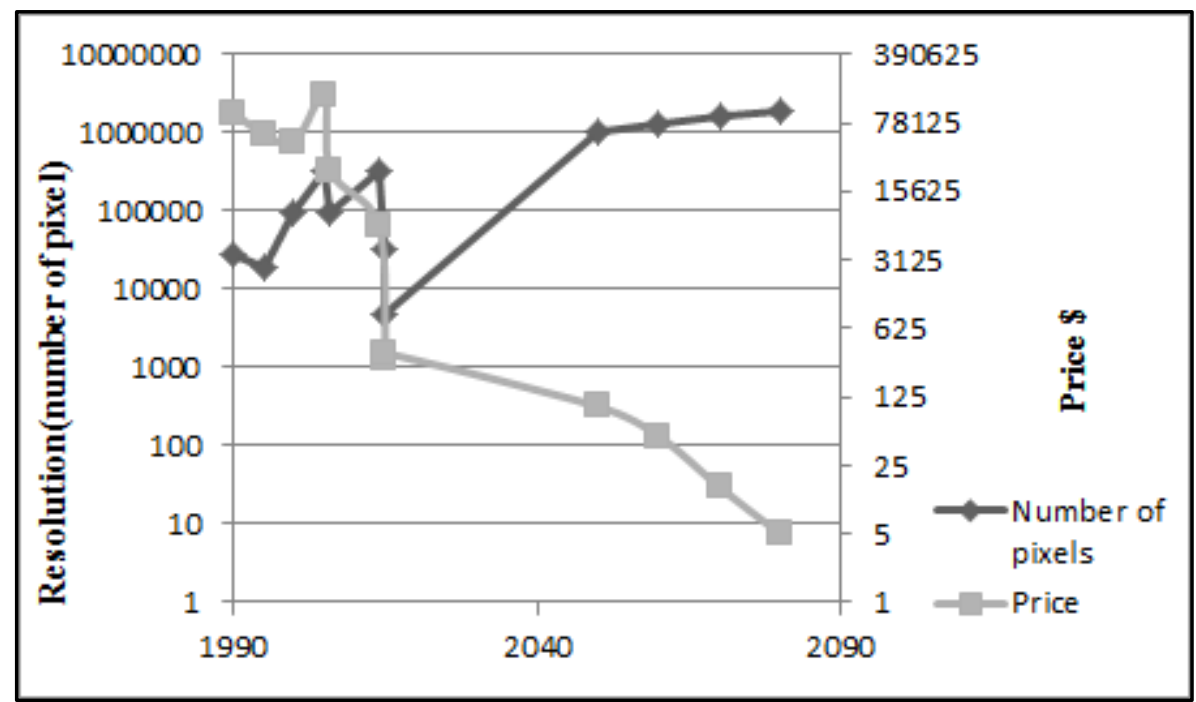

Fig. 4. Figure 4: Diagram of the changes of infrared camera cost and resolution from 1980 to 2060

\section{Conclusion}

In the future, with respect to detectors, IRT will continue to develop with higher resolution and lower cost due to the continuous development of uncooled FPAs (microbolometers) and cooled FPAs (photonics, quantum detectors for high-end applications). With regard to image processing, IRT has evolved from digital functional imaging to pattern analysis, detection and quantitative analysis. Further developments are expected as well especially since new processors will be able to handle more complex algorithms (such as real-time thermal quadrupoles manipulation or complex thermal models for instance). Internet of things will also revolutionize IRT with a widespread use of internet connected low-cost smart IR sensors distributed at many places for a given application, in NDT and other fields. These improvements in IRT will lead to a greater acceptance in industry and elsewhere (even by the general public) and a variety of "hot applications" will emerge. This will for instance include humanoid robots which will be fitted with IRT as well.

\section{REFERENCES}

[1] X. Maldague, "Theory and practice of infrared technology for nondestructive testing," 2001.

[2] J. Y. Andersson and A. Karim, "Infrared detectors: Advances, challenges and new technologies," in IOP Conference Series: Materials Science and Engineering, vol. 51, pp. 12001-12008, IOP Publishing, 2013.

[3] S. Ness, C. N. Sherlock, P. O. Moore, and P. Mclntire, Nondestructive testing overview. American Society for Nondestructive Testing, 1996.

[4] A. Rogalski, "Infrared detectors: status and trends," Progress in quantum electronics, vol. 27, no. 2, pp. 59-210, 2003.

[5] "About asnt," Introduction to Nondestructive Testing. https://www.asnt.org/MinorSiteSections/AboutASNT/Intro-toNDT. Web. 12 Apr. 2015.

[6] "Ndt and training," N. D. T. and NDT Online Training - 218-270-3182 - Hutchinson and Brainerd and MN. NDT and Training, N. D. T. and NDT Online Training - 218-270-3182 - Hutchinson and Brainerd and MN. http://trainingndt.com/. Web. 12 Apr. 2015.

[7] N. Avdelidis, T.-H. Gan, C. Ibarra-Castanedo, and X. Maldague, "Infrared thermography as a nondestructive tool for materials characterisation and assessment," in SPIE Defense, Security, and Sensing, pp. 801313-801313, International Society for Optics and Photonics, 2011.

[8] C. Corsi, "New frontiers for infrared," Opto-Electronics Review, vol. 23, no. 1, pp. 3-25, 2015.

[9] C. Corsi, "Infrared: a key technology for security systems," in Sensors, pp. 37-42, Springer, 2014.

[10] R. S. Riley, J. M. Ben-Ezra, D. Massey, R. L. Slyter, and G. Romagnoli, "Digital photography: a primer for pathologists," Journal of clinical laboratory analysis, vol. 18, no. 2, pp. 91-128, 2004.

[11] G. C. Righini et al., An introduction to optoelectronic sensors. World Scientific, 2009.

[12] C. Downs and T. E. Vandervelde, "Progress in infrared photodetectors since 2000," Sensors, vol. 13, no. 4, pp. 5054-5098, 2013. 
[13] V. Vavilov, "Thermal ndt: historical milestones, state-of-the-art and trends," Quantitative InfraRed Thermography Journal, vol. 11, no. 1, pp. 66-83, 2014.

[14] M. Yakushev, D. Brunev, V. Varavin, V. Vasilyev, S. Dvoretskii, I. Marchishin, A. Predein, I. Sabinina, Y. G. Sidorov, and A. Sorochkin, "HgCdTe heterostructures on Si (310) substrates for midinfrared focal plane arrays," Semiconductors, vol. 45, no. 3, pp. 385-391, 2011.

[15] A. Rogalski, Infrared detectors. CRC Press, 2010.

[16] G. Hyseni, N. Caka, and K. Hyseni, "Infrared thermal detectors parameters: semiconductor bolometers versus pyroelectrics," WSEAS Transactions on circuits and systems, vol. 9, no. 4, pp. 238-247, 2010.

[17] C. Corsi, "History highlights and future trends of infrared sensors," Journal of modern optics, vol. 57, no. 18, pp. 1663-1686, 2010.

[18] "What is an infrared detector?," What Is an Infrared Detector, Ir Detectors, Photodetector. http://www.futureelectronics.com/en/optoelectronics/infrared-detector.aspx.Web. 12 Apr. 2015.

[19] A. Rogalski, "History of infrared detectors," Opto-Electronics Review, vol. 20, no. 3, pp. 279-308, 2012.

[20] A. Rogalski, "Recent progress in infrared detector technologies," Infrared Physics \& Technology, vol. 54, no. 3, pp. 136-154, 2011.

[21] A. Rogalski, "Infrared detectors for the future," Acta Physica Polonica-Series A General Physics, vol. 116, no. 3, p. 389, 2009.

[22] A. Rogalski, J. Antoszewski, and L. Faraone, "Third-generation infrared photodetector arrays," Journal of Applied Physics, vol. 105, no. 9, p. 091101, 2009.

[23] W. L. Wolfe and P. W. Kruse, "Thermal detectors," OSA Handbook of Optics, 2004.

[24] A. Kylili, P. A. Fokaides, P. Christou, and S. A. Kalogirou, "Infrared thermography (irt) applications for building diagnostics: A review," Applied Energy, vol. 134, pp. 531-549, 2014.

[25] R. G. Driggers, "What's new in infrared systems?," SPIE Professional April, 2014.

[26] N. K. Dhar, A. K. Sood, and R. Dat, Advances in infrared detector array technology. INTECH Open Access Publisher, 2013.

[27] P. Martyniuk, J. Antoszewski, M. Martyniuk, L. Faraone, and A. Rogalski, "New concepts in infrared photodetector designs," Applied Physics Reviews, vol. 1, no. 4, p. 041102, 2014.

[28] S. Tsao and M. Razeghi, "Quantum dots," Photonics, Volume 2: Nanophotonic Structures and Materials, p. $169,2015$.

[29] G. W. Hunter, J. R. Stetter, P. J. Hesketh, and C.-C. Liu, "Smart sensor systems," The Electrochemical Society Interface, vol. 19, no. 4, p. 29, 2010.

[30] C. Corsi, "Smart sensors," Infrared physics \& technology, vol. 49, no. 3, pp. 192-197, 2007.

[31] R. Cook, "Smart infrared temperature sensors: Making sense of the new generation," Sensors, vol. 17, no. 11, pp. 48-55, 2000.

[32] C. Corsi, "Smart sensors: Why and when the origin was and why and where the future will be," in SPIE OPTO, pp. 899302-899302, International Society for Optics and Photonics, 2013.

[33] O. Kanoun and H.-R. Trankler, "Sensor technology advances and future trends," Instrumentation and Measurement, IEEE Transactions on, vol. 53, no. 6, pp. 1497-1501, 2004.

[34] N. K. Suryadevara and S. C. Mukhopadhyay, Smart Homes: Design, Implementation and Issues, vol. 14. Springer, 2015.

[35] M. N. Abedin, I. Bhat, S. D. Gunapala, S. V. Bandara, T. F. Refaat, S. P. Sandford, and U. N. Singh, "The future of single-to multi-band detector technologies: Review," 2006.

[36] "Terahertz technology continuous-wave thz generation," . Web. 2 May 2015. http://www.toptica.com/products/terahertz-generation/terahertz-technologysources-and-thz-generation-methods/cw-terahertz-generation.html.

[37] "Terahertz technology pulsed terahertz generation," http:/www.toptica.com/products/terahertz-generation/terahertztechnologysources-and-thz-generation-methods/pulsed-terahertz-generation.html.

[38] Y.-K. Zhu, G.-Y. Tian, R.-S. Lu, and H. Zhang, "A review of optical ndt technologies," Sensors, vol. 11, no. 8, pp. 7773-7798, 2011.

[39] A. Rogalski and F. Sizov, "Terahertz detectors and focal plane arrays," Opto-electronics review, vol. 19, no. 3, pp. 346-404, 2011.

[40] N. Karpowicz, H. Zhong, J. Xu, K.-I. Lin, J.-S. Hwang, and X. Zhang, "Comparison between pulsed terahertz timedomain imaging and continuous wave terahertz imaging," Semiconductor Science and Technology, vol. 20, no. 7, p. S293, 2005.

[41] C. Jansen, S. Wietzke, O. Peters, M. Scheller, N. Vieweg, M. Salhi, N. Krumbholz, C. Jördens, T. Hochrein, and M. Koch, "Terahertz imaging: applications and perspectives," Applied optics, vol. 49, no. 19, pp. E48-E57, 2010.

[42] G. Ok, H. J. Kim, H. S. Chun, and S.-W. Choi, "Foreign-body detection in dry food using continuous sub-terahertz wave imaging," Food Control, vol. 42, pp. 284-289, 2014.

[43] C. Pradere, J. Caumes, E. Palomo, and J. Batsale, "Use of svd decomposition to increase signal and noise ratio on thz imaging measurements.,"

[44] A. Abina, U. Puc, A. Jeglič, and A. Zidanšek, "Applications of terahertz spectroscopy in the field of construction and building materials," Applied Spectroscopy Reviews, vol. 50, no. 4, pp. 279-303, 2015.

[45] T. Hochrein, "Terahertz technology is advancing-change from pure scientific to commercial use observerd," 
[46] T. Nagatsuma, "Terahertz technologies: present and future," IEICE Electronics Express, vol. 8, no. 14, pp. 1127-1142, 2011.

[47] "Terahertz \& sub-thz imaging technology — terasense," http://terasense.com/technology. Web. 2 May 2015.

[48] S. I. Gubarev, A. A. Dremin, V. E. Kozlov, V. M. Muravev, and I. V. Kukushkin, "Plasma excitations in the twodimensional electron system with lateral screening by metallic gate," JETP Letters, 90, 588 (2009).

[49] "New terahertz imaging systems," http://www.nethis-thz.com/en/nethis-products/nethis-teracam. Web. 2 May 2015.

[50] B. S. Karasik, A. V. Sergeev, and D. E. Prober, "Nanobolometers for thz photon detection," Terahertz Science and Technology, IEEE Transactions on, vol. 1, no. 1, pp. 97-111, 2011.

[51] D. M. Mittleman, "Frontiers in terahertz sources and plasmonics," Nature Photonics, vol. 7, no. 9, pp. 666-669, 2013.

[52] C. Ibarra-Castanedo, J.-M. Piau, S. Guilbert, N. P. Avdelidis, M. Genest, A. Bendada, and X. P. Maldague, "Comparative study of active thermography techniques for the nondestructive evaluation of honeycomb structures," Research in Nondestructive Evaluation, vol. 20, no. 1, pp. 1-31, 2009.

[53] C. Ibarra-Castanedo, D. Gonzalez, M. Klein, M. Pilla, S. Vallerand, and X. Maldague, "Infrared image processing and data analysis," Infrared physics \& technology, vol. 46, no. 1, pp. 75-83, 2004.

[54] R. Hidalgo-Gato, J. Andrés, J. M. López-Higuera, and F. Madruga, "Quantification by signal to noise ratio of active infrared thermography data processing techniques," Optics and Photonics Journal, vol. 3, no. 04, p. $20,2013$.

[55] X. P. Maldague, "Introduction to ndt by active infrared thermography," Materials Evaluation, vol. 60, no. 9, pp. 1060-1073, 2002.

[56] X. Maldague, F. Galmiche, and A. Ziadi, "Advances in pulsed phase thermography," Infrared physics \& technology, vol. 43, no. 3, pp. 175-181, 2002.

[57] F. Lopez, C. Ibarra-Castanedo, X. Maldague, and V. Nicolau, "Pulsed thermography signal processing techniques based on the $1 \mathrm{~d}$ solution of the heat equation applied to the inspection of laminated composites," Mater. Eval, vol. 72 , pp. 91-102, 2014.

[58] C. Ibarra-Castanedo, Quantitative subsurface defect evaluation by pulsed phase thermography: depth retrieval with the phase. PhD thesis, Université Laval, 2005.

[59] C. Ibarra-Castanedo, D. Gonzalez, F. Galmiche, X. Maldague, and A. Bendada, "Discrete signal transforms as a tool for processing and analyzing pulsed thermographic data," in Defense and Security Symposium, pp. 620514-620514, International Society for Optics and Photonics, 2006.

[60] X. Maldague and S. Marinetti, "Pulse phase infrared thermography," Journal of Applied Physics, vol. 79, no. 5, pp. 2694-2698, 1996.

[61] F. Madruga, C. Ibarra-Castanedo, O. Conde, J. Lopez-Higuera, and X. Maldague, "Automatic data processing based on the skewness statistic parameter for subsurface defect detection by active infrared thermography," in Proc. QIRT, vol. 9, 2008.

[62] F. Lopez, C. Ibarra-Castanedo, X. Maldague, and V. de Paulo Nicolau, "Analysis of signal processing techniques in pulsed thermography," in SPIE Defense, Security, and Sensing, pp. 87050W-87050W, International Society for Optics and Photonics, 2013.

[63] H. D. Benitez, C. Ibarra-Castanedo, A. Bendada, X. Maldague, H. Loaiza, and E. Caicedo, "Defect quantification with reference-free thermal contrast and artificial neural networks," in Defense and Security Symposium, pp. 65410V-65410V, International Society for Optics and Photonics, 2007.

[64] H. Benítez, C. Ibarra-Castanedo, A. Bendada, X. Maldague, H. Loaiza, and E. Caicedo, "Modified differential absolute contrast using thermal quadrupoles for the nondestructive testing of finite thickness specimens by infrared thermography," in Electrical and Computer Engineering, 2006. CCECE'06. Canadian Conference on, pp. 1039-1042, IEEE, 2006.

[65] L. Bai, B. Gao, S. Tian, Y. Cheng, Y. Chen, G. Y. Tian, and W. Woo, "A comparative study of principal component analysis and independent component analysis in eddy current pulsed thermography data processing," Review of Scientific Instruments, vol. 84, no. 10, p. 104901, 2013.

[66] F. López, V. Nicolau, X. Maldague, and C. Ibarra-Castanedo, "Multivariate infrared signal processing by partial leastsquares thermography," Proceedings of the VIlth International Workshop Advances in Signal Processing for NDT,Destructive Evalution of Materials, Québec, Canada, 2013.

[67] J. Bin, F.-F. Ai, N. Liu, Z.-M. Zhang, Y.-Z. Liang, R.-X. Shu, and K. Yang, "Supervised principal components: a new method for multivariate spectral analysis," Journal of Chemometrics, vol. 27, no. 12, pp. 457-465, 2013.

[68] J. E. Ospina, J. F. Florez, H. D. Benitez, and X. Maldague, "Thermal diffusivity estimation with quantitative pulsed phase thermography," SPIE: Thermosense XXXVI, vol. 9485, 2015.

[69] "Uses, applications and hazards of infrared radiation," HubPages.http://hubpages.com/hub/Uses-Hazards-andApplications-of-Infrared-Radiation. Web. 12 Apr. 2015.

[70] "Future of ndt in uk economy," http://www.bindt.org/downloads/Materials-KTN-Future-of-NDT-in-UK-economy.pdf.Web. 12 Apr. 2015.

[71] G. Ds, P. Ripak, P. Cerckel, D. Degrève, M. Katz, P. Fontana, A. Xheneumont, and F. Vanseven, "Some applications of virtual instrumentation in ndt," Laborelec. University of Brussels. Disponible en: http://www. ulb. ac. be/polytech/laborulb/athens/papere. pdf[Links], 2001.

[72] P. Moghadam and S. Vidas, "Heatwave: the next generation of thermography devices," in SPIE Sensing Technology+ 
Applications, pp. 91050F-91050F, International Society for Optics and Photonics, 2014.

[73] S. Vidas and P. Moghadam, "Heatwave: A handheld 3d thermography system for energy auditing," Energy and Buildings, vol. 66, pp. 445-460, 2013.

[74] S. Vidas, P. Moghadam, and M. Bosse, "3d thermal mapping of building interiors using an rgb-d and thermal camera," in Robotics and Automation (ICRA), 2013 IEEE International Conference on, pp. 2311-2318, IEEE, 2013.

[75] C. Mineo, D. Herbert, M. Morozov, S. Pierce, P. Nicholson, and I. Cooper, "Robotic non-destructive inspection," in 51st Annual Conference of the British Institute of Non-Destructive Testing, pp. 345-352, 2012.

[76] E. Guizzo, "A robot in the family," Spectrum, IEEE, vol. 52, no. 1, pp. 29-58, 2015.

[77] M. Stolze, "Technology solutions and opportunities," The Measure of Innovation, 5. 2015.

[78] "Internet of thingswikipedia.org," wikipedia.org. 5.2015.

[79] "For your life," FLIR Systems. http://www.flir.ca. Web. 12 Apr. 2015.

[80] "Thermal imaging camera," Seek Thermal.http://www.thermal.com/. Web. 12 Apr. 2015. 